\title{
SOME REMARKS ON DENSITIES IN THE HEISENBERG GROUP
}

\author{
Valentino Magnani \\ Università di Pisa, Dipartimento di Matematica \\ Largo Pontecorvo 5, I-56127 Pisa, Italy; magnani@dm.unipi.it
}

\begin{abstract}
We observe that upper densities and spherical Federer densities may differ on all two dimensional surfaces of the sub-Riemannian Heisenberg group. This provides an entire class of intrinsic rectifiable sets having upper density strictly less than one.
\end{abstract}

\section{Introduction}

Area formulae in homogeneous groups are part of the wider project to develop Geometric Measure Theory in a non-Euclidean framework. Here a basic difficulty is the possible difference between Hausdorff dimension and topological dimension of smooth submanifolds. It turns out that such submanifolds cannot be rectifiable, according to the metric notion of rectifiability, [6]. This problem already appears in the Heisenberg group, which is the simplest noncommutative homogeneous group, see for instance [24].

Measure theoretic area formulae are the key tool to overcome the lack of Lipschitz parametrizations. If $\mathcal{S}^{\alpha}$ is the $\alpha$-dimensional spherical measure, $A \subset X$ is a Borel set of a metric space $X, \mathcal{S}^{\alpha}(A)<+\infty$ and $\mu\llcorner A$ is absolutely continuous with respect to $\mathcal{S}^{\alpha}\llcorner A$, then the following integration formula holds

$$
\mu(B)=\int_{B} \theta^{\alpha}(\mu, x) d \mathcal{S}^{\alpha}(x)
$$

for each Borel set $B \subset A$, where $\mu$ is a Borel regular measure on $X$. The previous formula also requires that $X$ is the countable union of open sets having $\mu$ finite measure, that for instance $(0,+\infty) \ni r \rightarrow \operatorname{diam}(\mathbf{B}(x, r))$ is continuous for all $x \in X$ and all metric balls with positive radius have positive diameter, see [16, Theorem 11]. The point is the explicit formula of the spherical Federer $\alpha$-density $\theta^{\alpha}(\mu, \cdot)$, namely,

(2) $\theta^{\alpha}(\mu, x)=\inf _{\varepsilon>0} \sup \left\{\frac{\mu(\mathbf{B})}{c_{\alpha} \operatorname{diam}(\mathbf{B})^{\alpha}}: x \in \mathbf{B}, \mathbf{B}\right.$ is a closed ball, $\left.\operatorname{diam}(\mathbf{B})<\varepsilon\right\}$,

where $c_{\alpha}>0$ can be arbitrarily fixed. This formula suggests that $\theta^{\alpha}(\mu, x)$ can be seen as a kind of "non-centered upper density", that may differ from the standard upper $\alpha$-density

$$
\Theta^{* \alpha}(\mu, x)=\limsup _{r \rightarrow 0^{+}} \frac{\mu(\mathbf{B}(x, r))}{c_{\alpha}(2 r)^{\alpha}} .
$$

We refer to 2.10 .19 of [6] for more information on upper and lower $\alpha$-densities in metric spaces. When they coincide, their common value is the $\alpha$-density, denoted by $\Theta^{\alpha}(\mu, \cdot)$.

https://doi.org/10.5186/aasfm.2017.4217

2010 Mathematics Subject Classification: Primary 28A75; Secondary 53C17, 22E30.

Key words: Heisenberg group, Hausdorff measure, density, rectifiability. 
In the important case of rectifiable measures, where $E \subset \mathbf{R}^{n}$ is $k$-rectifiable, $\mathcal{H}_{|\cdot|}^{k}$ is the Euclidean Hausdorff measure and setting $\mu=\mathcal{H}_{|\cdot|}^{k}\llcorner E$, we have

$$
\Theta^{k}\left(\mathcal{H}_{|\cdot|}^{k}\llcorner E, \cdot)=\theta^{k}\left(\mathcal{H}_{|\cdot|}^{k}\llcorner E, \cdot)=1\right.\right.
$$

$\mathcal{H}_{|\cdot|}^{k}$ a.e. in $E$, see for instance 3.2.19 of [6]. This implication holds for more general rectifiable sets in metric spaces, [11, Theorem 9]. Conversely, in Euclidean space it is well known that the validity of (3) a.e. implies the $k$-rectifiability of $E,[18$, Theorem 2.1]. Moreover, only the existence of the $k$-density a.e. implies the same $k$-rectifiability, [21]. A recent account on densities and rectifiability in the Euclidean space can be found in [4].

Natural notions of "intrinsic rectifiability" in Heisenberg groups and general stratified groups have also been studied, playing an analogous role to the classical rectifiability, $[7,8,15]$. Thus, we may ask to what extent we can expect to find deep relationships between rectifiability and densities in these groups.

A first promising result is by Mattila, Serapioni and Serra Cassano, who characterize the intrinsic rectifiability in Heisenberg groups by the a.e. existence of suitable tangent subgroups of fixed dimension, [19]. More recently, the Marstrand's density theorem in Heisenberg groups have been proved by Chousionis and Tyson, [3].

We focus our attention on one codimensional intrinsic rectifiable sets of the first Heisenberg group $\mathbf{H}$, which are called $\mathbf{H}$-rectifiable sets, see Definition 2.4. $\mathbf{H}$ rectifiable sets may be far from being $C^{1}$ smooth and form a strictly larger class of $C^{1}$ smooth surfaces, [1], [12]. Our question is the following: is it reasonable to expect (3) to persist also when $E$ is H-rectifiable? The following theorem answers the above question, when the sub-Riemannian distance $\rho$, in short SR distance, is fixed in $\mathbf{H}$.

Theorem 1.1. There exists a geometric constant $0<\gamma_{\rho}<1$, only depending on $\rho$, such that for each $\mathcal{S}_{\rho}^{3}$-measurable and $\mathbf{H}$-rectifiable set $\Sigma \subset \mathbf{H}$ with $\mathcal{S}_{\rho}^{3}(\Sigma)<+\infty$, we have

$$
\Theta^{* 3}\left(\mathcal{H}_{\rho}^{3}\llcorner\Sigma, x) \leq \Theta^{3}\left(\mathcal{S}_{\rho}^{3}\llcorner\Sigma, x)=\gamma_{\rho}\right.\right.
$$

for $\mathcal{S}_{\rho}^{3}$-a.e. $x \in \Sigma$, where $\mathcal{H}_{\rho}^{3}$ is the Hausdorff measure constructed by $\rho$.

Let us point out that the inequality in (4) is a simple consequence of the existence of $\Theta^{3}\left(\mathcal{S}_{\rho}^{3}\llcorner\Sigma, x)\right.$. Theorem 1.1 is strictly related to the shape of the metric unit ball $\mathbf{B}_{\rho}$, see (7). In fact, the geometric constant $\gamma_{\rho}$ is a quotient between the areas of two suitable slices of $\mathbf{B}_{\rho}$, according to (9), (10) and (18). Let us point out that the densities in (4) are independent of the constant $c_{3}$ of (9), that is used for both their definition and for the construction of the measures $\mathcal{H}_{\rho}^{3}$ and $\mathcal{S}_{\rho}^{3}$.

The estimate (4) shows that the SR distance is not suitable to develop analogues of the classical rectifiability theorems. On the other hand, a few intriguing questions are now in order. If we choose a different homogeneous distance $d$ of $\mathbf{H}$, see Definition 2.2, what can we say about the existence of the density $\Theta^{3}\left(\mathcal{H}_{d}^{3}\llcorner\Sigma, \cdot)\right.$ and its value? It is also unclear whether the upper density $\Theta^{* 3}\left(\mathcal{H}_{d}^{3}\llcorner\Sigma, \cdot)\right.$ is either one or strictly less than one $\mathcal{H}_{d^{3}}^{3}$ a.e. in $\Sigma$. The proof of Theorem 1.1 follows from the fact that the 3 -density for the spherical measure $\mathcal{S}_{\rho}^{3}\left\llcorner\Sigma\right.$ actually $\mathcal{S}_{\rho}^{3}$-a.e. exists and it is equal to $\gamma_{\rho}$. This is a consequence of the a.e. blow-up of the perimeter measure, see $\left[9\right.$, Theorem 3.1], and its integral representation with respect to $\mathcal{S}_{\rho}^{3}$, see $[17$, Theorem 1.3]. 
We wish to stress that the strict inequality $\gamma_{\rho}<1$ is possible due to the nonconvex shape of the metric ball $\mathbf{B}_{\rho}$. This special sub-Riemannian phenomenon could not appear in $\mathbf{R}^{n}$ equipped with the usual commutative operations, since here any homogeneous distance gives a Banach norm and all metric balls of a Banach space are convex. In fact, in any finite dimensional Banach space Theorem 1.1 cannot hold, as a consequence of [11, Theorem 9].

The estimate (4) also affects the relationship between the spherical measure $\mathcal{S}_{\rho}^{3}$ and the centered Hausdorff measure $\mathcal{C}_{\rho}^{3}$. The latter, especially known in Fractal Geometry, was introduced by Saint Raymond and Tricot, [23]. It is a variant of the spherical measure, that could be seen as "dual" of the packing measure, in view of its relationship to upper density as the packing measure has with its lower density, see [23, Theorem 1.1] and [5] for more information. We have the following theorem.

Theorem 1.2. For each $\mathbf{H}$-rectifiable set $\Sigma \subset \mathbf{H}$ such that $0<\mathcal{S}_{\rho}^{3}(\Sigma)<+\infty$, there holds

$$
\mathcal{S}_{\rho}^{3}(\Sigma)<\mathcal{C}_{\rho}^{3}(\Sigma)
$$

Let us point out that in general $\mathcal{S}_{\rho}^{3} \leq \mathcal{C}_{\rho}^{3}$. By contrast, in the same Heisenberg group, we have $\mathcal{S}_{\rho}^{4}=\mathcal{C}_{\rho}^{4}$, where 4 is the Hausdorff dimension of $\mathbf{H}$ and this fact extends to more general groups, [10, Corollary 4.13]. It is worth comparing Theorem 1.2 also with Euclidean results. In fact, here spherical measures and centered Hausdorff measures always coincide on rectifiable sets, see both Corollary 9.4 and Corollary 9.5 of [23]. It can be also observed that in the assumptions of Theorem 1.2, replacing $\rho$ by any homogeneous distance $d$ whose unit ball is convex, then $\mathcal{C}_{d}^{3}(\Sigma)=\mathcal{S}_{d}^{3}(\Sigma)$, see Remark 2.2. All of these facts show how Theorem 1.2 provides another unexpected feature of intrinsic rectifiable sets of $\mathbf{H}$, when seen through the SR distance.

Some additional questions are still to be understood. In fact, again in the case where the metric unit ball with respect to homogeneous distance $d$ is convex, then the same arguments for the proof of Theorem 1.1, joined with Theorem 5.2 of [17], imply that

$$
\Theta^{3}\left(\mathcal{S}_{d}^{3}\llcorner\Sigma, x)=1\right.
$$

for $\mathcal{S}_{d}^{3}$-a.e. $x \in \Sigma$, where $\Sigma$ is $\mathbf{H}$-rectifiable, see Remark 2.3. Since we may choose an $\mathbf{H}$-rectifiable set $\Sigma_{0}$ that is not rectifiable in the metric sense, [12], formula (6) shows the existence of a metric space $(\mathbf{H}, d)$ having an unrectifiable set $\Sigma_{0}$ whose 3 -density with respect to $\mathcal{S}_{d}^{3}\llcorner\Sigma$ is equal to one a.e. in $\Sigma$. To the author's knowledge, the question of finding a metric space along with a subset which is not $k$-rectifiable and whose $k$-density equals one a.e. is still an important open question when $k>1$. For $k=1$ this cannot happen and it is a consequence of the main theorem of [22]. The point in our case is that the 3-density refers to the 3-dimensional Hausdorff measure and the exact formula relating $\mathcal{S}_{d}^{3}\left\llcorner\Sigma\right.$ and $\mathcal{H}_{d}^{3}\llcorner\Sigma$ in $\mathbf{H}$ is still unknown, even for 2-dimensional smooth submanifolds.

For this reason, it is not yet clear how far the previous example is from the open question of finding suitable $k$-unrectifiable sets of a metric space, having $k$-density, with respect to the Hausdorff measure, that is equal to one a.e. and $k>1$. 


\section{Basic definitions and proofs}

We consider the three dimensional Heisenberg group $\mathbf{H}$, which is represented by $\mathbf{R}^{3}$ equipped with the Lie group operation

$$
(x, y, t) \cdot\left(x^{\prime}, y^{\prime}, t^{\prime}\right)=\left(x+x^{\prime}, y+y^{\prime}, t+t^{\prime}-2 x y^{\prime}+2 x^{\prime} y\right)
$$

for all $(x, y, t),\left(x^{\prime}, y^{\prime}, t^{\prime}\right) \in \mathbf{H}$ and the left invariant vector fields

$$
X=\partial_{x}+2 y \partial_{t}, \quad Y=\partial_{y}-2 x \partial_{t} \quad \text { and } \quad T=\partial_{t} .
$$

This system of coordinates also yields an auxiliary Euclidean structure on $\mathbf{H}$, where $|\cdot|$ denotes the Euclidean norm of $\mathbf{R}^{3}$, that is automatically induced on $\mathbf{H}$.

Now we introduce the distance that makes $\mathbf{H}$ an SR manifold. It is well known that $\mathbf{H}$ is pathwise connected by horizontal curves, namely absolutely continuous curves $\gamma:[0,1] \rightarrow \mathbf{H}$ such that $\dot{\gamma}(t)$ is a linear combination of $X(\gamma(t))$ and $Y(\gamma(t))$ for a.e. $t \in[0,1]$. This permits us to define the following SR distance

$$
\rho(p, q)=\inf \left\{\int_{0}^{1} \sqrt{\langle\dot{\gamma}(t), \dot{\gamma}(t)\rangle_{g}} d t: \gamma \text { is horizontal and connects } p \text { with } q\right\}
$$

between any couple of points $p, q \in \mathbf{H}$, where $\langle\cdot, \cdot\rangle_{q}$ denotes the left invariant Riemannian metric that makes the vector fields $X, Y, T$ everywhere orthonormal.

Explicit formulae for geodesics with respect to $\rho$ and the equations for the the boundary of the SR unit ball

$$
\mathbf{B}_{\rho}=\{p \in \mathbf{H}: \rho(p, 0) \leq 1\}
$$

are well known facts, see for instance [20] and [2]. Precisely, the boundary of $\mathbf{B}_{\rho}$ is the image of the mapping

$$
\Phi:[0,2 \pi] \times[-2 \pi, 2 \pi] \rightarrow \mathbf{R}^{3}
$$

whose components are defined as follows

$$
\left\{\begin{array}{l}
\Phi_{1}(\theta, \varphi)=\frac{\cos \theta(1-\cos \varphi)+\sin \theta \sin \varphi}{\varphi} \\
\Phi_{2}(\theta, \varphi)=\frac{-\sin \theta(1-\cos \varphi)+\cos \theta \sin \varphi}{\varphi} \\
\Phi_{3}(\theta, \varphi)=2\left(\frac{\varphi-\sin \varphi}{\varphi^{2}}\right)
\end{array}\right.
$$

where we understand $\Phi_{1}(\theta, 0)=\sin \theta, \Phi_{2}(\theta, 0)=\cos \theta$ and $\Phi_{3}(\theta, 0)=0$. The precise shape of the unit ball $\mathbf{B}_{\rho}$ will play an important role in the subsequent computation.

The distance $\rho$ allows us to construct the associated spherical measure and Hausdorff measure. These measures can be constructed in a general metric space.

Definition 2.1. (Hausdorff measures) Let $X$ be a metric space equipped with a distance $d$. Let $\mathcal{F} \subset \mathcal{P}(X)$ be a nonempty class of closed subsets of $X$, let $\alpha>0$ and $c_{\alpha}>0$. If $\delta>0$ and $E \subset X$, then we define

$$
\phi_{\delta}(E)=\inf \left\{\sum_{j=0}^{\infty} c_{\alpha} \operatorname{diam}\left(B_{j}\right)^{\alpha}: E \subset \bigcup_{j \in \mathbf{N}} B_{j}, \operatorname{diam}\left(B_{j}\right) \leq \delta, B_{j} \in \mathcal{F}\right\} .
$$

When $\mathcal{F}$ is the family of closed balls, then the $\alpha$-dimensional spherical measure is defined by $\mathcal{S}^{\alpha}(E)=\sup _{\delta>0} \phi_{\delta}(E)$, for every $E \subset \mathbf{G}$. When $\mathcal{F}$ is the family of closed subsets, then the $\alpha$-dimensional Hausdorff measure is defined by $\mathcal{H}^{\alpha}(E)=$ $\sup _{\delta>0} \phi_{\delta}(E)$. In the case $c_{\alpha}=2^{-\alpha}$, we will use the notation $\mathcal{S}_{0}^{\alpha}$ and $\mathcal{H}_{0}^{\alpha}$. 
If in the previous definition the distance $d$ coincides with the SR distance $\rho$,

$$
c_{3}=\beta_{\rho} 2^{-3} \text { and } \quad \beta_{\rho}=\max _{w \in \mathbf{B}_{\rho}} \mathcal{H}_{|\cdot|}^{2}\left(\mathbf{B}_{\rho} \cap w N_{0}\right),
$$

where $N_{0}=\left\{(x, 0, z) \in \mathbf{R}^{3}\right\}$, then the corresponding spherical measure and Hausdorff measure are denoted by $\mathcal{S}_{\rho}^{3}$ and $\mathcal{H}_{\rho}^{3}$, respectively. We also introduce

$$
\beta_{\rho}^{0}=\mathcal{H}_{|\cdot|}^{2}\left(\mathbf{B}_{\rho} \cap N_{0}\right) .
$$

A more general class of distances than $\rho$ can be considered in $\mathbf{H}$, as shown in the next definition.

Definition 2.2. (Dilations and homogeneous distances) For every $s>0$, we define the dilation $\delta_{s}: \mathbf{H} \rightarrow \mathbf{H}$ as

$$
\delta_{s}(x, y, z)=\left(s x, s y, s^{2} z\right) \quad \text { for every }(x, y, z) \in \mathbf{H} .
$$

A distance $d$ in $\mathbf{H}$ is homogeneous if it is continuous, and for each $q, w, u \in \mathbf{H}$ and $r>0$ we have $d(q w, q u)=d(w, u)$ and $d\left(\delta_{r} w, \delta_{r} u\right)=r d(w, u)$.

It is well known that $\rho$ is a homogeneous distance, see for instance [13].

Definition 2.3. (Centered Hausdorff measure) Let $X$ be a metric space equipped with a distance $d$. We fix $\alpha>0$ and $c_{\alpha}>0$ and denote by $\mathcal{F}_{b}$ the family of closed balls in $X$. For any $\delta>0$ and $S \subset X$, we define

$\mathcal{C}_{\delta}^{\alpha}(S)=\inf \left\{\sum_{j=0}^{\infty} c_{\alpha} \operatorname{diam}\left(B_{j}\right)^{\alpha}: S \subset \bigcup_{j \in \mathbf{N}} B_{j}, B_{j} \in \mathcal{F}_{b}\right.$ is centered in $\left.S, \operatorname{diam}\left(B_{j}\right) \leq \delta\right\}$.

Thus, we set $\tilde{\mathcal{C}}^{\alpha}(S)=\sup _{\delta>0} \mathcal{C}_{\delta}^{\alpha}(S)$ and for $E \subset X$ we define

$$
\mathcal{C}^{\alpha}(E)=\sup \left\{\tilde{\mathcal{C}}^{\alpha}(S): S \subset E\right\} \text {. }
$$

According to [23, Lemma 3.1], $\mathcal{C}^{\alpha}$ is a Borel regular outer measure, called the $\alpha$ dimensional centered Hausdorff measure. When $c_{\alpha}=2^{-\alpha}$, we use the notation $\mathcal{C}_{0}^{\alpha}$.

Let us point out that $\tilde{\mathcal{C}}^{\alpha}$ may not be a measure. In fact, an example of [23, Section 4], shows that it is not monotone. The following standard inequalities also hold

$$
\mathcal{H}^{\alpha} \leq \mathcal{S}^{\alpha} \leq \mathcal{C}^{\alpha} \leq 2^{\alpha} \mathcal{H}^{\alpha} .
$$

For a 3-dimensional centered Hausdorff measure $\mathcal{C}_{d}^{3}$, which is constructed by a homogeneous distance $d$ of $\mathbf{H}$, we use the same constant $c_{3}=\beta_{\rho} 2^{-3}$ of the spherical measure $\mathcal{S}_{\rho}^{3}$.

Definition 2.4. (H-rectifiable set and $\mathbf{H}$-regular set) We say that $S \subset \mathbf{H}$ is $\mathbf{H}$-rectifiable if for each $j \in \mathbf{N}$ there exists a function $f_{j}: \Omega_{j} \rightarrow \mathbf{R}$, where $\Omega_{j} \subset \mathbf{H}$ is an open set, such that the directional derivatives

$$
\Omega_{j} \ni x \rightarrow X f_{j} \text { and } \Omega \ni x \rightarrow Y f_{j}
$$

are continuous, the mapping $\Omega_{j} \ni x \rightarrow(X f, Y f)$ is everywhere nonvanishing and

$$
\mathcal{H}_{\rho}^{3}\left(S \backslash \bigcup_{j=1}^{\infty} f_{j}^{-1}(0)\right)=0 .
$$

The sets $f_{j}^{-1}(0)$ are called $\mathbf{H}$-regular. 
Remark 2.1. Notice that the defining functions $f_{j}$ in the previous definition may not be necessarily differentiable in the classical sense, even on a set of positive measure, [14].

2.1. Proof of Theorem 1.1. Following notation and definitions of [17], $\Sigma$ is contained in a countable union of portions of reduced boundaries of $h$-finite perimeter sets, up to an $\mathcal{S}_{\rho}^{3}$ negligible set. By our assumptions on $\Sigma$, standard arguments allow us to assume that

$$
\Sigma=\Omega \cap \mathcal{F}_{H} E,
$$

without any loss of generality. In the previous formula, $\Omega \subset \mathbf{H}$ is an open set, $E \subset \mathbf{H}$ is a set of $h$-finite perimeter and the reduced boundary $\mathcal{F}_{H} E$ intersected with $\Omega$ coincides with $\Omega \cap \partial E$, that is an $\mathbf{H}$-regular set. Since $\mathbf{H}$-regular sets are obviously $\mathbf{H}$-rectifiable, Theorem 1.2 of [17] gives

$$
\left|\partial_{H} E\right|\left\llcorner\Omega=\beta\left(\rho, \nu_{E}\right) \mathcal{S}_{0}^{3}\left\llcorner\left(\Omega \cap \mathcal{F}_{H} E\right)=\beta\left(\rho, \nu_{E}\right) \mathcal{S}_{0}^{3}\llcorner\Sigma\right.\right.
$$

and for each $x \in \Sigma$ there holds

$$
\beta\left(\rho, \nu_{E}(x)\right)=\max _{z \in \mathbf{B}_{\rho}} \mathcal{H}_{|\cdot|}^{2}\left(\mathbf{B}_{\rho}(z, 1) \cap N\left(\nu_{E}(x)\right)\right)
$$

where $N\left(\nu_{E}(x)\right)$ is the vertical plane orthogonal to the generalized inward normal $\nu_{E}(x)$, see for instance [17] for more information on these notions.

The rotational symmetry of $\mathbf{B}_{\rho}$ follows from (8) and it implies that $\beta(\rho, \cdot)$ is constant. Taking into account that translations preserve the Euclidean Hausdorff measure $\mathcal{H}_{|\cdot|}^{2}$ between translated vertical planes, it follows that

$$
\beta_{\rho}=\beta(\rho, \cdot),
$$

where $\beta_{\rho}$ is defined in (9). For the same reasons, the function

$$
v \rightarrow \mathcal{H}_{|\cdot|}^{2}\left(\mathbf{B}_{\rho} \cap N(v)\right)=\beta_{\rho}^{0}
$$

is independent of $v$ as it varies in $\mathbf{R}^{2} \times\{0\}$ and $\beta_{\rho}^{0}$ is defined in (10). As a result, by (11), we get $\left|\partial_{H} E\right|\left\llcorner\Omega=\mathcal{S}_{\rho}^{3}\left\llcorner\Sigma\right.\right.$. Since $\Omega \cap \mathcal{F}_{H} E=\Sigma$, we can apply Theorem 3.1 of [9] at any point $x \in \Sigma$, hence obtaining that

$$
\lim _{r \rightarrow 0^{+}} \frac{\left|\partial_{H} E\right|(\mathbf{B}(x, r))}{r^{3}}=\mathcal{H}_{|\cdot|}^{2}\left(\mathbf{B}_{\rho} \cap N_{0}\right)=\beta_{\rho}^{0} .
$$

It follows that

$$
\begin{aligned}
\Theta^{* 3}\left(\mathcal{S}_{\rho}^{3}\llcorner\Sigma, x)\right. & =\limsup _{r \rightarrow 0^{+}} \frac{\left|\partial_{H} E\right|(\mathbf{B}(x, r))}{\beta_{\rho} r^{3}}=\lim _{r \rightarrow 0^{+}} \frac{\left|\partial_{H} E\right|(\mathbf{B}(x, r))}{\beta_{\rho} r^{3}} \\
& =\Theta^{3}\left(\mathcal{S}_{\rho}^{3}\llcorner\Sigma, x)=\frac{\beta_{\rho}^{0}}{\beta_{\rho}} .\right.
\end{aligned}
$$

Finally, our claim follows by checking that $\beta_{\rho}^{0}<\beta_{\rho}$. This is a direct computation that can be carried out by using equations (8). Since $\Phi_{1}^{2}(\theta, \varphi)+\Phi_{2}^{2}(\theta, \varphi)=(2-2 \cos \varphi) / \varphi^{2}$ one realizes that the intersection of $\partial \mathbf{B}_{\rho}$ with $\{(x, 0, z): x, z \geq 0\}$ can be parametrized by the curve

$$
\tilde{\Phi}(\varphi)=\left(\frac{\sqrt{2-2 \cos \varphi}}{\varphi}, 0, \frac{2 \varphi-2 \sin \varphi}{\varphi^{2}}\right)
$$


defined on $[0,2 \pi]$, where we understand $\tilde{\Phi}(0)=(1,0,0)$. It follows that the closed upper half of $\partial \mathbf{B}_{\rho}$ is the image of the mapping $F:[0,2 \pi] \times[0,2 \pi] \rightarrow \mathbf{R}^{3}$ defined as

$$
\left\{\begin{array}{l}
F_{1}(\psi, \varphi)=\frac{\sqrt{2-2 \cos \varphi}}{\varphi} \cos \psi, \\
F_{2}(\psi, \varphi)=\frac{\sqrt{2-2 \cos \varphi}}{\varphi} \sin \psi, \\
F_{3}(\psi, \varphi)=2\left(\frac{\varphi-\sin \varphi}{\varphi^{2}}\right)
\end{array}\right.
$$

where $F_{1}(\psi, 0)=\cos \psi, F_{2}(\psi, 0)=\sin \psi$ and $F_{3}(\psi, 0)=0$. From both the rotational and the antipodal symmetry of $\mathbf{B}_{\rho}$, if we set $A_{\rho}=\mathbf{B}_{\rho} \cap\left\{(x, 0, z) \in \mathbf{R}^{3}: x, y \geq 0\right\}$ and take into account (10), then Gauss-Green's theorem applied to the curve $\tilde{\Phi}$ of (13) gives

$$
\frac{\beta_{\rho}^{0}}{4}=\mathcal{H}^{2}\left(A_{\rho}\right)=2 \int_{0}^{2 \pi} \frac{\sqrt{2-2 \cos \varphi}}{\varphi^{4}}(2 \sin \varphi-\varphi \cos \varphi-\varphi) d \varphi .
$$

Intersecting $\mathbf{B}_{\rho}$ with the subset

$$
S_{1}=\left\{(x, y, z) \in \mathbf{R}^{3}: y=2 \sqrt{2} / 3 \pi, x, z \geq 0\right\},
$$

we get the curve $\tilde{F}:[0,3 \pi / 2] \rightarrow \mathbf{R}^{3}$, defined as

$$
\tilde{F}(\varphi)=\left(\sqrt{\frac{2-2 \cos \varphi}{\varphi^{2}}-\frac{8}{9 \pi^{2}}}, \frac{2 \sqrt{2}}{3 \pi}, \frac{2 \varphi-2 \sin \varphi}{\varphi^{2}}\right),
$$

where we understand $\tilde{F}(0)=\left(\sqrt{1-8 / 9 \pi^{2}}, \frac{2 \sqrt{2}}{3 \pi}, 0\right)$. In fact, the function

$$
\varphi \rightarrow \frac{1-\cos \varphi}{\varphi^{2}}
$$

is decreasing on $(0,2 \pi)$, it vanishes on $3 \pi / 2$ and the image of $\tilde{F}$ is the set

$$
S_{1} \cap \partial \mathbf{B}_{\rho} .
$$

Thus, setting $A_{\rho}^{1}=\mathbf{B}_{\rho} \cap S_{1}$, Gauss-Green's theorem gives

$$
\mathcal{H}_{|\cdot|}^{2}\left(A_{\rho}^{1}\right)=2 \int_{0}^{\frac{3 \pi}{2}} \sqrt{\frac{2-2 \cos \varphi}{\varphi^{2}}-\frac{8}{9 \pi^{2}}}\left(\frac{2 \sin \varphi-\varphi \cos \varphi-\varphi}{\varphi^{3}}\right) d \varphi,
$$

where introducing the plane

$$
N_{1}=\left\{(x, y, z) \in \mathbf{R}^{3}: y=2 \sqrt{2} / 3 \pi\right\},
$$

the rotational symmetry of $\mathbf{B}_{\rho}$ gives

$$
\mathcal{H}_{|\cdot|}^{2}\left(\mathbf{B}_{\rho} \cap N_{1}\right)=4 \mathcal{H}_{|\cdot|}^{2}\left(A_{\rho}^{1}\right) \text {. }
$$

Using for instance the computer program Maple, one can verify that the integral in (15) is strictly less than the integral in (17), hence

$$
\gamma_{\rho}=\frac{\beta_{\rho}^{0}}{\beta_{\rho}} \leq \frac{\beta_{\rho}^{0}}{\mathcal{H}_{|\cdot|}^{2}\left(\mathbf{B}_{\rho} \cap N_{1}\right)}<1 .
$$

This concludes the proof of Theorem 1.1.

2.2. Proof of Theorem 1.2. The arguments of [10, Theorem 4.28] allow us to replace $\mathcal{S}_{\rho}^{3}\left\llcorner\Sigma\right.$ by the perimeter measure $\left|\partial_{H} E\right|\llcorner\Omega$, for an $h$-finite perimeter set 
$E \subset \mathbf{H}$, with no loss of generality. The measure theoretic area formula (1.4) of [10] and Theorem 3.1 of [9], joined with Theorem 1.2 of [17], give the equalities

$$
\left|\partial_{H} E\right|\left\llcorner\Omega=\beta_{\rho}^{0} \mathcal{C}_{\rho}^{3}\left\llcorner\Sigma=\beta_{\rho} \mathcal{S}_{\rho}^{3}\llcorner\Sigma .\right.\right.
$$

In view of (18), we have

$$
\mathcal{S}_{\rho}^{3}(\Sigma)=\frac{\beta_{\rho}^{0}}{\beta_{\rho}} \mathcal{C}_{\rho}^{3}(\Sigma)<\mathcal{C}_{\rho}^{3}(\Sigma),
$$

hence concluding the proof.

Remark 2.2. It may be surprising that despite the validity of Theorem 1.2, the equality $\mathcal{S}_{d}^{3}\left\llcorner\Sigma=\mathcal{C}_{d}^{3}\left\llcorner\Sigma\right.\right.$ holds when $d=d_{\infty}$ and $\Sigma$ is a general H-rectifiable set. This is proved in [10] where this formula is obtained for G-rectifiable sets in a stratified group $\mathbf{G}$ and the dimension of the measures is $Q-1$, where $Q$ is the Hausdorff dimension of the group. Indeed, the previous formula can be extended to every homogeneous distance $d$, whose unit ball is a convex set with respect to the linear structure assumed on the group, namely

$$
\mathcal{S}_{d}^{Q-1}\left\llcorner\Sigma=\mathcal{C}_{d}^{Q-1}\llcorner\Sigma\right.
$$

where $\Sigma \subset \mathbf{G}$ is a $\mathbf{G}$-rectifiable set. This can be proved following the same arguments of [10], joined with Theorem 5.2 of [17].

Remark 2.3. The same arguments in the proof of Theorem 1.1 can be carried out with respect to any another homogeneous distance. As a consequence, by Theorem 5.2 of [17] the ratio in (12) becomes equal to one, where $\rho$ is replaced by a homogeneous distance whose unit ball is a convex set.

Acknowledgements. It is my pleasure to thank Aleksandra Zapadinskaya for her kind contribution in finding the areas of slices of the sub-Riemannian unit ball. I am also grateful to Francesco Serra Cassano for his useful comments.

\section{References}

[1] Balogh, Z. M.: Size of characteristic sets and functions with prescribed gradient. - J. Reine Angew. Math. 564, 2003, 63-83.

[2] Bellaïche, A.: The tangent space in sub-Riemannian geometry. - In: Sub-Riemannian geometry, Progr. Math. 144, Birkhäuser, Basel, 1996, 1-78.

[3] Chousionis, V., and J. T. Tyson: Marstrand's density theorem in the Heisenberg group. Bull. Lond. Math. Soc. 47:5, 2015, 771-788.

[4] De Lellis, C.: Rectifiable sets, densities and tangent measures. - Zur. Lect. Adv. Math., Eur. Math. Soc., Zürich, 2008.

[5] Edgar, G. A.: Centered densities and fractal measures. - New York J. Math. 13:2, 2007, $33-87$.

[6] Federer, H.: Geometric measure theory. - Springer, 1969.

[7] Franchi, B., R. Serapioni, and F. Serra Cassano: Justifiability and perimeter in the Heisenberg group. - Math. Ann. 321:3, 2001, 479-531, 2001.

[8] Franchi, B., R. Serapioni, and F. Serra Cassano: Regular hypersurfaces, intrinsic perimeter and implicit function theorem in Carnot groups. - Comm. Anal. Geom. 5, 2003, 909-944.

[9] Franchi, B., R. Serapioni, and F. Serra Cassano: On the structure of finite perimeter sets in step 2 Carnot groups. - J. Geom. Anal. 13:3, 2003, 421-466. 
[10] Franchi, B., R. P. Serapioni, and F. Serra Cassano: Area formula for centered Hausdorff measures in metric spaces. - Nonlinear Anal. 126, 2015, 218-233.

[11] Kirchneim, B. Rectifiable metric spaces: local structure and regularity of the Hausdorff measure. - Proc. Amer. Math. Soc. 121:1, 1994, 113-123.

[12] Kirchneim, B., and F. Serra Cassano: Rectifiability and parameterization of intrinsic regular surfaces in the Heisenberg group. - Ann. Sc. Norm. Super. Pisa Cl. Sci. (5) 3:4, 2004, $871-896$.

[13] Magnani, V.: Elements of geometric measure theory on sub-Riemannian groups. - PhD thesis, Scuola Normale Superiore, Pisa, 2002.

[14] Magnani, V.: The coarea formula for real-valued Lipschitz maps on stratified groups. - Math. Nachr. 278:14, 2005, 1689-1705.

[15] Magnani, V.: Characteristic points, rectifiability and perimeter measure on stratified groups. - J. Eur. Math. Soc. 8:4, 2006, 585-609.

[16] Magnani, V. On a measure theoretic area formula. - Proc. Roy. Soc. Edinburgh Sect. A 145, $2015,885-891$.

[17] Magnani, V.: A new differentiation, shape of the unit ball and perimeter measure. - Indiana Univ. Math. J. (to appear).

[18] Mattila, P.: Hausdorff $m$ regular and rectifiable sets in $n$-space. - Trans. Amer. Math. Soc. 205, 1975, 263-274.

[19] Mattila, P., R. Serapioni, and F. Serra Cassano: Characterizations of intrinsic rectifiability in Heisenberg groups. - Ann. Sc. Norm. Super. Pisa Cl. Sci. (5) 9:4, 2010, 687-723.

[20] Monti, R.: Some properties of Carnot-Carathéodory balls in the Heisenberg group. - Atti Accad. Naz. Lincei Cl. Sci. Fis. Mat. Natur. Rend. Lincei (9) Mat. Appl. 11:3, 2000, 155-167.

[21] Preiss, D.: Geometry of measures in $\mathbf{R}^{n}$ : distribution, rectifiability, and densities. - Ann. of Math. (2) 125:3, 1987, 537-643.

[22] Preiss, D., and J. TišER: On Besicovitch's $\frac{1}{2}$-problem. - J. London Math. Soc. (2) 45:2, 1992, 279-287.

[23] Saint Raymond, X., and C. Tricot: Packing regularity of sets in $n$-space. - Math. Proc. Cambridge Philos. Soc. 103:1, 1988, 133-145.

[24] Stein, E. M.: Harmonic analysis: real-variable methods, orthogonality, and oscillatory integrals. - Princeton Math. Ser. 43, Princeton Univ. Press, Princeton, NJ, 1993.

Received 17 February 2016• Accepted 16 August 2016 